IUPAC Technical Report

Hitoshi Ishida*, Jean-Claude Bünzli and Andrew Beeby

\title{
Guidelines for measurement of luminescence spectra and quantum yields of inorganic and organometallic compounds in solution and solid state (IUPAC Technical Report)
}

DOI 10.1515/pac-2014-0706

Received July 10, 2014; accepted July 29, 2016

\begin{abstract}
Guidelines for measuring the luminescence of inorganic compounds, metal complexes, and organometallic compounds are described. Common textbooks and manuals describing luminescence measurements are usually targeted for organic compounds, and are not always suitable for inorganic and organometallic compounds, which emit room-temperature phosphorescence. The report describes problems that researchers may confront while recording emission data and elaborates clear procedures to avoid these problems and provide adequate standardized protocols.
\end{abstract}

Keywords: IUPAC inorganic chemistry division; luminescence spectra; measurement procedures - lifetimes; phosphorescence spectra; quantum yields; standards.

\section{CONTENTS}

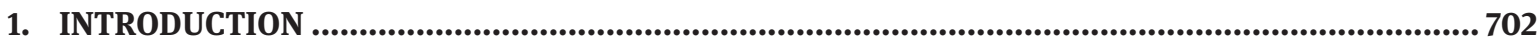

2. PHOTOPHYSICAL PROPERTIES OF INORGANIC COMPOUNDS ................................................702

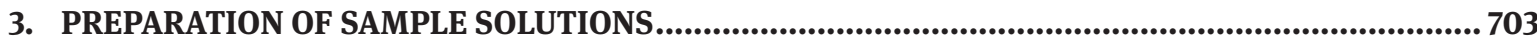

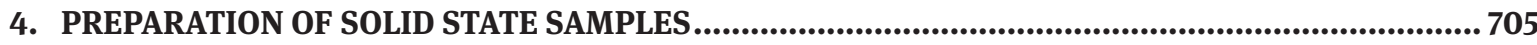

5. INSTRUMENTS FOR MEASURING LUMINESCENCE SPECTRA ......................................................705

6. MEASUREMENT OF QUANTUM YIELDS IN SOLUTION ................................................................706

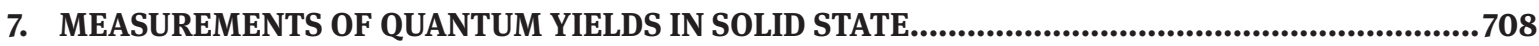

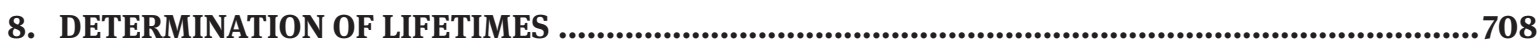

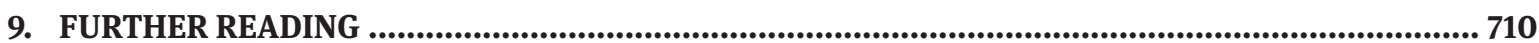

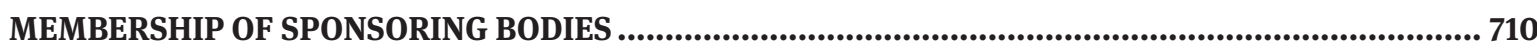

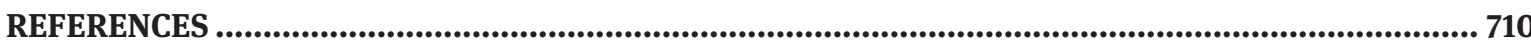

\footnotetext{
*Corresponding author: Hitoshi Ishida, Department of Chemistry, Graduate School of Science, Kitasato University, 1-15-1 Kitasato, Minami-ku, Sagamihara, Kanagawa 252-0373, Japan, e-mail: ishida@sci.kitasato-u.ac.jp Jean-Claude Bünzli: Swiss Federal Institute of Technology Lausanne (EPFL) Institute of Chemical Sciences and Engineering BCH 1402, CH-1015 Lausanne, Switzerland and Haimen Institute of Science and Technology, Hong Kong Baptist University, Hong Kong, SAR, P.R. China

Andrew Beeby: Department of Chemistry, Durham University, South Road, Durham DH1 3LE, UK
} 


\section{Introduction}

Recent progress in electroluminescent devices and luminescent molecular probes has led chemists to design and synthesize novel emissive compounds, materials, and hybrid materials $[1,2]$. The photophysical properties of newly synthesized inorganic compounds are commonly measured using commercially available luminescence spectrometers, much as is done for absorption spectra. However, most luminescence spectrometers are single channel instruments and spectra have to be corrected for the instrumental function, which is a fact largely ignored by non-specialists. Commercial instruments are often not designed or optimized for the study of materials with unusual properties, for example compounds that emit in the near infrared (NIR) spectral region or solid state samples that are often encountered in inorganic materials. Furthermore, experimentalists need to be aware that minute quantities of highly luminescent impurities (e.g. those present in the solvent - sometimes as a stabilizer) can lead to erroneous data. When measuring weakly luminescent samples, the resulting spectra may be contaminated by spurious bands from the instrumental set-up, such as ghosts bands from the gratings or coatings of the mirrors, second-order Rayleigh scattering bands, Raman bands from the solvent, luminescent impurities in the glass/quartz cuvettes or capillaries (e.g. chromium), or even contamination of the sample holder by careless users. Therefore it is difficult even for well-trained photochemists to estimate the reliability of luminescence data, unless careful blank experiments and standard measurements are performed. The exquisite sensitivity of luminescence spectroscopy is sometimes its downfall and great care needs to be taken when determining photophysical properties.

Accordingly, projects concerning photoluminescence measurements have been carried out with the support of the IUPAC Organic and Biomolecular Chemistry, Physical and Biophysical Chemistry, and Analytical Chemistry Divisions [3-5]. Although they are valuable and important projects, the relevant reports are not entirely appropriate for inorganic chemists who encounter specific problems linked to the materials under investigation. In addition, these reports are written by specialists in photochemistry who have a strong physical background. Synthetic inorganic chemists would benefit from more detailed and specific guidelines for the measurements of luminescence spectra, lifetimes, and quantum yields of inorganic and organometallic compounds, since these materials have intrinsic properties differing considerably from those of organic compounds.

We therefore propose procedures for measuring emission spectra, quantum yields and lifetimes of inorganic compounds, metal complexes, and organometallic compounds both in solution and in the solid state. Attention will be given to draft the protocols as clearly as possible so that inorganic chemists newly entering into the field can reliably get the photophysical parameters they need. Measurements of solid-state samples are much more difficult than solutions so that, we also give suggestions for this type of experiments.

\section{Photophysical properties of inorganic compounds}

Photochemistry textbooks mostly describe emission properties of organic compounds, not inorganic ones [6-9]. It is important to understand the difference between the luminescence of organic and inorganic compounds [10, 11].

An organic compound absorbing light is usually promoted to excited singlet states. In most organic compounds, the emission arises from the first excited singlet state. It is a fast decaying process between two states with same spin and it is called "fluorescence" since $\Delta S=0$. The textbooks or laboratory manuals for photochemistry generally deal with this aspect of luminescence. The characteristics of fluorescence are as follows: (i) The wavelength at absorption maximum is close to the fluorescence maximum (typically $20-50 \mathrm{~nm}$ ). The difference between the band maxima of the absorption and emission spectra is called "Stokes' shift". For a compound with a small Stokes' shift, the emission band may overlap somewhat with the absorption band, therefore re-absorption of the emitted light becomes a problem. The effects of 
re-absorption, referred to as "inner filter effect" are a distortion of the observed spectrum, particularly at the short wavelength edge, a reduction in the observed fluorescence quantum yield, and possibly, a change in the emission lifetime [12, 13]. The textbooks recommend keeping the sample concentration very low, advising that the absorbance at the excitation wavelength should be $<0.05$ [4]. This also ensures validity of the Beer-Lambert law and minimizes inner filter effects. (ii) The lifetimes of the singlet excited states are short, typically in the nanosecond time range and, accordingly, the singlet excited states are not easily quenched by oxygen dissolved in the solvent.

In a luminescent inorganic compound, light can be absorbed directly by the metal center, by the ligand, or by a metal-ligand based transition. In the first case, molar absorption coefficients are usually small since electric-dipole $\mathrm{d}-\mathrm{d}$ and $\mathrm{f}-\mathrm{f}$ transitions are forbidden by Laporte's rule. Therefore, most of the time, light is absorbed by the surrounding ligands that, as for organic compounds, are promoted into a singlet excited state. This state can emit light, but due to the presence of a heavy element such a metal ion, spin-orbit coupling, which increases with increasing atomic number, easily converts the excitation energy to triplet states. Emission from this state can often be observed even at room temperature in solution. According to IUPAC definition, phosphorescence is a spin-forbidden radiative transition (i.e. $\Delta S>0$ ); $\Delta S=1$ for triplet state emission [14]. The characteristics of phosphorescence are as follows: (i) The absorption and emission maxima are well separated and the large Stokes' shift implies that re-absorption is less important compared to fluorescence; (ii) The lifetimes of the triplet excited states are relatively long (tens of nanoseconds to milliseconds), and accordingly the triplet excited states are more sensitive to quenching by oxygen.

In the case of lanthanide ions, energy can be directly funneled from the singlet state into one of the higher energy multiplet state of the metal ion or transferred to such a state from the ligand triplet state(s). The resulting metal-centered luminescence is called "sensitized luminescence" and the associated shift between excitation and emission is termed "ligand-induced Stokes' shift" [15]. The excitation scheme described above is oversimplified since charge transfer and d-f states are also often implied in excitation/emission spectra, particularly in compounds with d-transition metal ions. But the final outcome, i.e. emission from a multiplet state of the metal center or of a metal-ligand moiety, is the same. Metal-centered emission is often a phosphorescence process $(\Delta S>0)$, although some transitions may well correspond to a fluorescence process $(\Delta S=0)$; as a result it is preferable to use the generic term "luminescence" when describing these spectra (or "photoluminescence" if excitation is achieved by light). Emission from inorganic compounds is in many cases temperature-dependent because of the presence of higher-energy and/or non-emissive states, which are thermally accessible.

\section{Preparation of sample solutions}

It is vital that all glassware is kept meticulously clean; solvents must be of spectroscopic grade and checked, in any case, for background fluorescence and cutoff wavelength: trusting the label indications is not recommended.

Sample purity: The sample must be of the highest possible purity. Luminescent impurities prevent the observation of accurate emission spectra, while non-luminescent impurities may quench the excited state of the sample, resulting in decreased intensities of the emission bands and/or in a different sensitization mechanism. In addition, non-luminescent impurities which absorb at the excitation wavelength could affect emission quantum yield determination.

Important tests for sample purity and homogeneity are:

(i) The emission spectrum is normally independent of the excitation wavelength, which can be tested by recording the emission at several wavelengths. In some instances though, when compounds display emissions from multiple origins, luminescence spectra may depend on the excitation wavelength even if the sample is pure; this is the case, for instance, with polynuclear copper(I), platinum(II) and gold(I) complexes [16-20]. 
(ii) The profile of the luminescence excitation spectrum should match the absorption spectrum. Any deviation from these conditions warrants detailed examination to ensure the observations are a very unusual property of the sample, rather than due to an impurity or dissociation of the sample. If the sample is a mixture, band intensities change with excitation wavelength. However, even if the sample is pure, aggregation in solution may alter the emission spectrum; therefore measuring the emission spectra with different concentrations of the sample is recommended.

Another major problem encountered with metal complexes, particularly with those that are labile, is partial dissociation of the ligand(s). This point is largely overlooked in most of the luminescence studies reported in the literature and the problem is severe, particularly when using luminescence as an analytical tool. Indeed, in this case, one has to keep the absorbance of the sample low in order to be in the linear range of Beer-Lambert law and of the luminescence experiment. Note that for a Metal(M):Ligand(L) complex with 1:1 stoichiometry and $\log _{10} K_{1}=10$ (the stability constant $K_{\mathrm{n}}$ is equal to $a\left(\mathrm{ML}_{\mathrm{n}}\right) /\left\{a(\mathrm{M})(a(\mathrm{~L}))^{\mathrm{n}}\right\}$ where quantities $a$ are the activities of the species in parentheses), a nanomolar solution contains the following mol\% species: $73 \%$ of the complex and $27 \%$ of the dissociated ligand. For a $1: 3$ complex with $\log _{10} K_{1}=8, \log _{10} K_{2}=6$, and $\log _{10} K_{3}=4$, the speciation for a micromolar solution is $41 \%$ (1:1), $58 \%$ (1:2), and $0.8 \%$ (1:3) while free ligand accounts for only $0.3 \%$; if the concentration is reduced to nanomolar, then the corresponding speciation is $22 \% 1: 1$ complex and $78 \%$ free ligand. There are three ways of determining to what extent the complex dissociates (it always dissociates to some extent):

(i) The best is of course to determine stability constants by spectrophotometric, potentiometric, or other spectroscopic titrations, and to calculate the corresponding speciation.

(ii) In absence of $\log _{10} K$ values, the best way is to prepare at least four dilutions of the sample spanning at least one order of magnitude (e.g. 0.1-1 $\mu \mathrm{M}$ ), as well as a blank; then both absorption and emission spectra are measured, and integrated emission intensities versus absorbances are reported on a graph: in the absence of substantial dissociation, the relationship is linear and the slope is the quantum yield.

(iii) Close comparison between the (corrected) excitation and the absorbance spectra is recommended. These two spectra exactly match when no dissociation occurs, the excitation spectra are corrected for the instrumental function, and experiments are conducted under conditions for which the Beer-Lambert law is valid. The good match between the two spectra also suggests that the metal-centered luminescence occurs from sensitization of the ligand(s). However, this method is not always reliable if there is only a small difference between the excitation/absorption spectra of the ligand and of the complex, which is often the case. Moreover it is certainly not capable, in most cases, of determining dissociation smaller than $10 \%$.

Selection of solvent: The solvent is selected so that it does not undergo reactions with irradiated materials and the sample dissolves satisfactorily without decomposition or dissociation; it should also fully transmit light at both excitation and emission wavelengths. Photochemists often use spectroscopy-grade water, methanol or acetonitrile because of their transparency.

Sample concentration: Ideally, sample concentration should be low enough so that the absorbance at the excitation wavelength is $<0.05$ to avoid concentration quenching problems and to keep within the linearity range of Beer-Lambert's law and luminescence experiment. We note that the larger Stokes shift associated with phosphorescence means that re-absorption is less of a problem for phosphorescence compared with fluorescence. Lowering the concentration may render the accurate estimation of absorbance at the excitation wavelength more difficult and, most importantly, could lead to dissociation of metal complexes, so that often a compromise has to be found. It is recommended to check the linearity of the emission intensities against the absorbance at the excitation wavelength. If the absorbance of the sample is $>0.5$, a correction for the innerfilter effect should be applied when calculating the quantum yield, a process that needs detailed knowledge of the spectrometer's optical layout [21].

De-aeration: For measuring room-temperature phosphorescence or sensitized luminescence through a triplet state, removal of oxygen by de-aeration or degassing is very important. The preferred method for 
sample degassing is the use of the freeze-pump-thaw method, for which up to 5 cycles using high vacuum $\left(<10^{-1} \mathrm{~Pa}\right)$ may be needed. If this is not possible, it is recommended to bubble $\mathrm{He}$, Ar or $\mathrm{N}_{2}$ through the sample via a septum for more than $20 \mathrm{~min}$, and then to measure the luminescence spectrum as soon as possible. With this method it is also essential to check the absorption spectrum as bubbling may cause evaporation of the solvent and hence concentration of the solution; the problem may be minimized by incorporating a bubbler containing the pure solvent upstream in the degassing gas line.

Temperature: In general, the emission from inorganic compounds strongly depends on the temperature. If possible, the temperature of the sample solution should be kept constant by using a thermostat. In any case, the temperature at which the sample is measured has to be reported.

\section{Preparation of solid state samples}

For measurements of luminescent properties of solid state samples, the sample purity is extremely important, because the quenching efficiencies and re-absorption effects can be rather higher than those observed in solutions. Therefore all details pertaining to the preparation and purification of the sample should be described so that results can be reproduced independently.

Solid samples should be thoroughly dried before sample preparation and, whenever relevant, the absence of water (or solvation) molecules checked by vibrational spectroscopy [22]. Two types of solid-state samples can be considered: powdered samples and thin films. Both can be measured by means of an integrating sphere.

The photophysical properties of powdered samples, particularly the absorption of light and emission intensity, depend on the size of the particles in the powder. Therefore, the samples should be thoroughly ground so as to obtain a very fine powder. Ideally, the samples should be sieved, but this is rarely done.

The photophysical properties of thin solid films are highly sensitive to the refractive index of the materials (i.e. in case of doping, to the doping concentration) that can induce wave-guiding effects and to the orientation of the emitting molecules within the film, which may lead to emission anisotropy. Therefore it is essential to make use of an integrating sphere that overcomes the latter problem. The thickness of the thin film should be constant and, whenever possible, determined, as should the exact concentration of the dopant. Moreover, in the case of doped thin films, several doping concentrations should be tested since it has often been reported that the quantum yield varies with the dopant concentration. Furthermore, when studying the luminescence quantum yield of a thin film doped with an emitter, the edges of the sample should be obscured to eliminate the contribution of the wave-guided light to the observed emission spectrum. The spectrum of the wave-guided light is particularly sensitive to the re-absorption of light in the film and varies with the distance from the point of illumination to the edge of the film.

\section{Instruments for measuring luminescence spectra}

Prior to taking measurements, the operator should understand the working principles of the spectrometer. Most popular systems are fitted with a scanning monochromator and a photomultiplier tube (PMT) detector. Alternatively, the system may be equipped with a spectrograph and a multichannel detector, such as a charge-coupled device (CCD) camera. A first important point is to verify the spectral response of the spectrometer and to establish, where options are available, which diffraction grating and/or detector is most suitable for the planned experiments. Instrument manufacturers normally provide two options for the PMT: one for ultraviolet (UV)/shorter visible wavelengths and another, a red-sensitive tube, for longer wavelengths. If emission at $\lambda>700 \mathrm{~nm}$ is to be measured, the second type must be selected. Single point detectors are available for the NIR spectral region, including silicon avalanche diodes (up to $1100 \mathrm{~nm}$ ), and a limited number of PMTs have sensitivity up to $1700 \mathrm{~nm}$, but these are quite expensive and have high dark-signal levels. Most 
CCD cameras are based upon silicon, giving them a useful wavelength range up to $1100 \mathrm{~nm}$, but in recent years NIR-CCDs based upon InGaAs have become available. In any case, the instrumental function of the spectrometer (i.e. its response versus wavelength) should be determined with an officially calibrated standard light source according to specific procedures described in the manuals provided by the manufacturers. It is also recommended to measure the emission spectra of accepted standards and to compare them with reported spectra in the literature [23].

There are four ways of reporting emission spectra:

(i) the number of emitted photons per second, unit area and wavelength [nm], $N_{\mathrm{em}}(\lambda)\left[\mathrm{s}^{-1} \mathrm{~m}^{-2} \mathrm{~nm}^{-1}\right]$

(ii) the number of emitted photons per second, unit area and wavenumber $\left[\mathrm{cm}^{-1}\right], N_{\mathrm{em}}(\tilde{v})\left[\mathrm{s}^{-1} \mathrm{~m}^{-2}\left(\mathrm{~cm}^{-1}\right)^{-1}\right]$

(iii) the irradiance per wavelength $[\mathrm{nm}],\left(E(\lambda)\left[\mathrm{W} \mathrm{m}^{-2} \mathrm{~nm}^{-1}\right]\right)$

(iv) the irradiance per wavenumber $\left[\mathrm{cm}^{-1}\right], E(\tilde{v})\left[\mathrm{W} \mathrm{m}^{-2}\left(\mathrm{~cm}^{-1}\right)^{-1}\right]$

The emission spectra are normally reported by normalizing the emission intensity at the maximum wavelength. The reporter should note that the maximum wavelength (or wavenumber) in the spectrum based on the number of photons is different from the one based on the irradiance, because the number of photons with a constant irradiance increases at longer wavelengths (or lower wavenumbers). When using a manufacturer's correction data the user should ask the instrument manufacturer whether the emission intensity is based on the numbers of photons or the irradiance. As described in the next section, the emission quantum yields relate to the number of photons and therefore the spectra with the emission intensity based on the number of photons are recommended. The user should also be aware that a scanning monochromator provides an approximately constant bandwidth in wavelength across the spectrum. Some manufacturers provide correction curves in energy per bandwidth and time, which is inappropriate since the user requires the intensity in quanta per bandwidth and time.

\section{Measurements of quantum yields in solution}

The emission quantum yield $(\Phi)$ is defined as the ratio of the number of photons emitted $\left(N_{\mathrm{em}}\right)$ to the number of photons absorbed $\left(N_{\mathrm{abs}}\right)$ (eq. 1) $[5,8,10]$.

$$
\Phi=\frac{N_{\text {em }}}{N_{\text {abs }}}
$$

Some instruments dedicated to the determination of luminescence quantum yields are presently commercially available. However, most laboratories still use conventional spectrofluorimeters which can give excellent quality data, provided that data are properly corrected for the spectral response of the instrument. Note that fully corrected emission spectra must be used and the emission intensity integrated over the entire emission spectral range. If the spectra with emission intensity based on the number of photons are obtained, integration can be performed directly:

$$
N_{\mathrm{em}}=\int_{0}^{\infty} N_{\mathrm{em}}(\lambda) \mathrm{d} \lambda=\int_{0}^{\infty} N_{\mathrm{em}}(\tilde{v}) \mathrm{d} v
$$

As the emission spectra represent the irradiance per wavelength in many common spectrometers, it should be converted to the number of photons by eq. 3:

$$
N_{\mathrm{em}}=\int_{0}^{\infty} \frac{\lambda}{h c} E(\lambda) \mathrm{d} \lambda=\int_{0}^{\infty} \frac{\lambda}{h c} E(\tilde{v}) \mathrm{d} v
$$

where $h$ is Planck's constant and $c$ is the speed of light.

Note that measurements should be repeated at least three times and, if possible, on more than two independently prepared samples so as to ensure good statistics. It is difficult to determine quantum 
yields with accuracy better than $10 \%$. There are three different ways of determining quantum yields of solutions:

(i) The first is described in Section 3, point (ii) and is interesting, since it allows one to simultaneously test if dissociation occurs or not. In principle, no standard is required, but it is nevertheless recommended to measure a compound with known quantum yield under the same experimental conditions (concentration, excitation wavelength, same range of emission spectrum) to test the instrument and the procedure.

(ii) The relative method that makes use of standard compounds is still frequently performed. The emission spectrum of the sample is compared to that of a recognized standard with quantum yield $\Phi_{\mathrm{s}}$, and the quantum yield $\Phi_{\mathrm{x}}$ of the sample is estimated with eq. 4:

$$
\Phi_{\mathrm{x}}=\Phi_{\mathrm{s}} \times\left(\frac{I(\mathrm{em})_{\mathrm{x}}}{I(\mathrm{em})_{\mathrm{s}}}\right) \times\left(\frac{A(\mathrm{ex})_{\mathrm{s}}}{A(\mathrm{ex})_{\mathrm{x}}}\right) \times\left(\frac{I(\mathrm{ex})_{\mathrm{s}}}{I(\mathrm{ex})_{\mathrm{x}}}\right) \times\left(\frac{n_{\mathrm{x}}}{n_{\mathrm{s}}}\right)^{2}
$$

where subscripts " $\mathrm{x}$ " and "s" are for the unknown sample and the standard, respectively; $I(\mathrm{em})$ is the integrated intensity of emission; $A(\mathrm{ex})$ is the absorbance at excitation wavelength; $I(\mathrm{ex})$ is the intensity of the excitation light; and $n$ is the refractive index of the solution (not the solvent; although if the solution is diluted, both values are very close). In an ideal case, the standard has to be selected so that the same excitation wavelength can be used for it and the sample and so that it emits in the same wavelength interval as the sample. If the emission spectrum consists of narrow emission bands, as for f-element ions, it is recommended to use a secondary standard consisting of a compound of the same metal ion. If the excitation wavelength for the sample is the same as the one for the standard, the correction for the intensity of the excitation light becomes unnecessary, reducing errors. The same solvent is also recommended for the measurements of the sample and the standard, in order to avoid the correction for the refractive indices (n). Therefore in this ideal case eq. 4 reduces to eq. 5.

$$
\Phi_{\mathrm{x}}=\Phi_{\mathrm{s}} \times\left(\frac{I(\mathrm{em})_{\mathrm{x}}}{I(\mathrm{em})_{\mathrm{s}}}\right) \times\left(\frac{A(\mathrm{ex})_{\mathrm{s}}}{A(\mathrm{ex})_{\mathrm{x}}}\right)
$$

It is furthermore recommended to prepare the two solutions with the same absorbance: if $A(\mathrm{ex})_{\mathrm{s}}=A(\mathrm{ex})_{\mathrm{x}}<0.5$, no correction is needed:

$$
\Phi_{\mathrm{x}}=\Phi_{\mathrm{s}} \times\left(\frac{I(\mathrm{em})_{\mathrm{x}}}{I(\mathrm{em})_{\mathrm{s}}}\right)
$$

On the other hand, if $A(\mathrm{ex})_{\mathrm{s}} \neq A(\mathrm{ex})_{\mathrm{x}}$, absorbances $A$ must be replaced with (1-10 $\left.0^{-A}\right)$ to take into account differences in the fraction of light absorbed. Finally, it is also recommended to use two different standards and to measure one standard against the other.

(iii) The absolute method, which makes use of an integration sphere [24] and which has long been in use by physicists and materials scientists, is not yet well known to chemists [25]. The integration sphere can be fitted on most commercial spectrofluorimeters as an accessory and has to conform to specific requirements. Unfortunately these are not always met in commercial spheres proposed by instrument manufacturers. The sample is put into a 2-3 mm I. D. quartz capillary. The method requires the measurement of (i) $L_{\mathrm{a}}$, the integrated intensity of light exiting the sphere when the empty capillary is illuminated at the excitation wavelength (Rayleigh scattering band); (ii) $L_{c}$, the same integrated intensity at the excitation wavelength when the sample is introduced into the sphere; these two measurements often necessitate the use of attenuators (transmission $0.01-10 \%$ ); (iii) $E_{\mathrm{c}}$, the integrated intensity of the entire emission spectrum. The absolute quantum yield is then given by:

$$
Q_{\mathrm{ab}}=\frac{E_{\mathrm{c}}}{\left[L_{\mathrm{a}}\left(\lambda_{\mathrm{ex}}\right)-L_{\mathrm{c}}\left(\lambda_{\mathrm{ex}}\right)\right] F_{\mathrm{att}}\left(\lambda_{\mathrm{ex}}\right)}
$$


whereby $F_{\text {att }}\left(\lambda_{\text {ex }}\right)$ is the correction for the attenuators used. The true transmission of the attenuators should be determined: do not trust manufacturers' datasheets. Reproducible and accurate data can be obtained when the fraction of absorbed light $\alpha=\left(L_{\mathrm{a}}-L_{\mathrm{c}}\right) / L_{\mathrm{a}}$ is in the range 0.10-0.90 [26]; both solutions (minimum volume: $\sim 60 \mu \mathrm{L}$ ) and powdered samples can be measured. Although the method does not need a standard, it is recommended to measure a compound with known quantum yield in parallel to test the procedure and the instrumentation.

Note on reference materials: In the literature, the three standards most used for quantum yield determination of both organic and inorganic compounds are quinine bisulfate, fluorescein, and Rhodamine 6G. However, care must be exerted. For instance, the quantum yield of quinine bisulfate is highly dependent upon the excitation wavelength, varying by as much as $50 \%$ between 280 and $380 \mathrm{~nm}$, so that excitation at $300 \mathrm{~nm}$ must be used to match quantum yields reported in the literature. Verification of the standards for inorganic compounds, such as metal complexes and organometallic compounds, is under way. For example, the quantum yields of $\left[\mathrm{Ru}(\mathrm{bpy})_{3}\right]^{2+}$, which is one of the most popular standards, has recently been re-evaluated as 0.063(3) in water and 0.095(3) in acetonitrile when excited at the metal-to-ligand charge transfer (MLCT) band around $450 \mathrm{~nm}$ under de-aerated conditions at $298 \mathrm{~K}$ [23, 25]. The value in aerated water, which is frequently used as an emission standard in biological studies, has also been reevaluated to be 0.040 . Other emissive metal complexes and organometallics are under examination.

\section{Measurements of quantum yields in solid state}

Progress in the study of nanomaterials has triggered a rapid increase in the number of reports describing luminescent properties of solid-state samples. This is probably related to the development of new instruments for measurements of the emission in solid state and driven by increased applications for solid-state emissive materials. Measurements of fluorescence from organic compounds have pointed out some problems: the nature of the solid samples, crystals or powder, strongly affects quantum yields, while structural defects in the crystals reduce quantum yields [25, 27]. Even the amount of sample used (e.g. in an integrating sphere) may affect the results. It is still uncertain whether similar problems will show up with inorganic compounds. However, polymorphs of the samples have been shown to present different optical properties [28, 29], and it is therefore recommended that the experimental conditions are reported in as much details as possible. The method of choice for such samples is the integration sphere and some wavelength-converting compounds (referred to as "phosphors" in the specialized literatures, e.g. $\mathrm{Y}_{2} \mathrm{O}_{3}: \mathrm{Eu}^{\mathrm{III}}$ or $\mathrm{BaMgAl}_{10} \mathrm{O}_{17}: \mathrm{Eu}^{\mathrm{II}}(\mathrm{BAM})$ ) or sodium salicylate (SA) are reasonable standards. In some cases, e.g. BAM or SA, correction should be made for reabsorption by the sample [30].

The use of an integrating sphere for quantum yield determinations has recently gained popularity. Building upon the widely cited method by Greenham and de Mello, new instrumentation and updated methods allow a range of sample types to be measured and the effects of re-absorption addressed [31-35]. Note that the same precautions used for solutions regarding sample purity and control experiments have to be taken to ensure reliable data.

\section{Determination of lifetimes}

The emission lifetimes of inorganic materials vary considerably and values commonly fall in a wide range, from nanoseconds to milliseconds, and, in some cases, seconds or even minutes. When lifetimes are short, $<100 \mathrm{~ns}$, time-correlated single photon counting (TCSPC) or high-frequency phase modulation methods have to be employed. When lifetimes are longer, measurements can be carried out using either photon counting with a multi-channel analyzer or a detector and oscilloscope combination. 
Samples for the determination of lifetimes should be prepared similarly to those for the determination of quantum yields. Again, attention should be given to the speciation in solution and to solvation of solid-state samples.

The following points have to be taken into consideration when performing the experiment:

(i) The signal level should be kept below saturation (i.e. it should lie in the linear range of the detector response, e.g. $<10^{6}$ count per second in photon counting mode with a PMT).

(ii) The time-window should be adjusted so that the entire profile is recorded, including the emission buildup, which can provide information on the energy transfer mechanism.

(iii) The decay should be measured until the signal reaches the background level; this means recording data for a period equal to at least five lifetimes.

(iv) Enough experimental points over the time-window should be recorded, typically $>100$, preferably 1000 if possible.

(v) For a simply emissive system the luminescence decay normally follows a single, first order process and the following equation is used:

$$
I(t)=A \cdot \mathrm{e}^{-k t}+B
$$

where $A$ is the pre-exponential factor, $k=1 / \tau$ is the observed decay rate constant, and $B$ is the background noise. The quality of fit is determined using an established statistical method, e.g. chi-square, and should have random weighted residuals. In many solid-state samples more complex decay functions may be observed. Understanding these can provide important information regarding the mechanism by which the final, emissive excited state is formed. One case is when decays can be fitted to a sum of exponentials:

$$
I(t)=\sum A_{i} e^{-k_{i} t}+B
$$

In this case, the populations $P_{i}$ of the different emitting sites can be evaluated as:

$$
P_{i}=\frac{\left(A_{i} / k_{i}\right)}{\sum_{i}\left(A_{i} / k_{i}\right)}=\frac{A_{i} \tau_{i}}{\sum_{i} A_{i} \tau_{i}} \text { with } \sum_{i} P_{i}=1
$$

while the intensity mean lifetime is:

$$
\tau_{\text {mean }}^{I}=\frac{\sum_{i}\left(P_{i} / k_{i}^{2}\right)}{\sum_{i}\left(P_{i} / k_{i}\right)}=\frac{\sum_{i} P_{i} \tau_{i}^{2}}{\sum_{i} P_{i} \tau_{i}}
$$

This lifetime has to be used when calculating the average collisional quenching constant within the frame of Stern-Volmer theory. In all other cases it is best to consider the amplitude mean lifetime:

$$
\tau_{\text {mean }}^{A}=\sum P_{i} \cdot \tau_{i}
$$

For some inorganic materials, the decays cannot be fitted with exponential functions and a "stretched exponential function" is used:

$$
I(t)=A \cdot e^{-(k t)^{\beta}} \text { with } \quad 0 \leq \beta \leq 1
$$

When non-single exponential kinetics are encountered, the physical origin of the decay should be thoroughly explored and satisfactorily explained [8].

(vi) The experiment should be repeated 3-5 times on 2-3 different samples, with a blank sample measured. 


\section{Further reading}

Photochemistry and photophysics of organic molecules is largely covered in the literature; as a start, we recommend reading some of these entries [36-38] as well as protocols for absolute determination of fluorescence quantum yields [39]. This guide to the expression of uncertainty in measurements is also important [40]. When it comes to inorganic compounds, the present guideline should be a helpful complement. Please note that measurement procedures for solid state samples still lack consistency. The methodology is expected to develop in the near future.

\section{Membership of sponsoring bodies}

Membership of the IUPAC Inorganic Chemistry Division Committee for the period 2012-2015 was as follows:

Division II Members 2012-2013

President: Loss, Robert D.; Vice President: Reedijk, Jan; Secretary: Leskela, Markku; Titular members: Mathur, Sanjay; Drabik, Milan; Sakai, Ken; Holden, Norman E.; Ohrstrom, Lars R.; Karen, Pavel; Tshuva, Edit Y.

Associate members: Ding, Tiping, Garcia-Martinez, Javier, Buchweishaija, Joseph; Rabinovich, Daniel; Vannier, Rose-Noelle; Kilic, Adem; National representatives: Abdul Aziz, Farina; Trendafilova, Natasha; Prugovečki, Biserka; Chandrasekhar, V.; Youngme, Sujittra; Toma, Henrique; Ali Saqib.

Division II Members 2014-2015

President: Reedijk, Jan; Vice President: Öhrström, Lars Secretary: Leskelä, Markku; Past President: Loss, Robert D.,

Titular members: Ding, Tiping, Drabik, Milan; Tshuva, Edit Y. Rabinovich, Daniel; Walczyk, Thomas; Wieser, Michael.

Associate members: Garcia-Martinez, Javier, Buchweishaija, Joseph; Vannier, Rose-Noelle; Kiliç, Adem; Sakai, Ken; Karen, Pavel; National representatives: Abdul Aziz, Farina; Armelao, Lidia; Badshah, Amin; Chandrasekhar, V.; Galamba Correia, João; Kalmykov, Stepan; Trendafilova, Natasha; Prugovečki, Biserka; Gabrolle Meesuk, Ladda; Mathur, Sanjay.

This document was prepared in the frame of IUPAC Project \#2009-045-1-200, Guidelines for Measurement of Luminescence Spectra and Quantum Yields of Inorganic Compounds, Metal Complexes and Materials.

Chair: Ishida, Hitoshi; Members: Beeby, Andrew; Bünzli, Jean-Claude G.; Campagna, Sebastiano; De Cola, Luisa; Ford, Peter; Gordon, Keith; Hasegawa, Yasuchika; Hasegawa, Miki; Katoh, Ryuzi; Keene, Richard; McCusker, James; Nozaki, Koichi; Sakai, Ken; Tobita, Seiji; Vlcek, Antonin; Yam, Vivian.

\section{References}

[1] S. Lamansky, P. Djurovich, D. Murphy, F. Abdel-Razzaq, H.-E. Lee, C. Adachi, P. E. Burrows, S. R. Forrest, M. E. Thompson. J. Am. Chem. Soc. 123, 4304 (2001).

[2] W. Brutting, C. Adachi, R. J. Holmes. Physics of Organic Semiconductors, 2nd., Wiley-VCH, New York (2012).

[3] A. M. Brouwer. Pure Appl. Chem. 83, 2213 (2011).

[4] U. Resch-Genger, P. C. DeRose. Pure Appl. Chem. 82, 2315 (2010).

[5] U. Resch-Genger, P. C. DeRose. Pure Appl. Chem. 84, 1815 (2012).

[6] N. J. Turro, J. C. Scaiano, V. Ramamurthy. Principles of Modern Molecular Photochemistry: An Introduction, University Science Books, Sausalito, CA (2008).

[7] N. J. Turro. Modern Molecular Photochemistry, University Science Books, Sausalito, CA (1991).

[8] J. Lakowicz, ed. Principles of Fluorescence Spectroscopy, 3rd ed. Springer, New York (2006).

[9] D. F. Eaton. Pure Appl. Chem. 60, 1107 (1988).

[10] M. Montalti, A. Credi, L. Prodi, M. T. Candolfi, eds. Handbook of Photochemistry, 3rd ed. CRC Press, Florida (2006).

[11] V. Balzani, P. Ceroni, A. Juris. Photochemistry and Photophysics: Concepts, Research, Applications, Wiley-VCH, New York (2014). 
[12] A. Kobe, A. Babnik, J. I. Mozina. in Proc. SPIE 3099, Micro-optical Technologies for Measurement, Sensors, and Microsystems II and Optical Fiber Sensor Technologies and Applications, O. M. Parriaux, E.-B. Kley, B. Culshaw, M. Breidne, (Eds.), pp. 369-375 (1997); https://spie.org/Publications/Proceedings/Paper/10.1117/12.281249.

[13] J. B. Birks. in Photophysics of Aromatic Molecules, pp. 92-93, Wiley, New York (1970).

[14] W. H. Melhuish. Pure Appl. Chem. 56, 231 (1984).

[15] S. I. Weissman. J. Chem. Phys. 10, 214 (1942).

[16] P. C. Ford, E. Cariati, J. Bourassa. Chem. Rev. 99, 3625 (1999).

[17] M. Fetterolf, A. E. Friedman, Y. Y. Yang, H. Offen, P. C. Ford. J. Phys. Chem. 92, 3760 (1988).

[18] V. W.-W. Yam, K. K.-W. Lo. Chem. Soc. Rev. 28, 323 (1999).

[19] V. W.-W. Yam, E. C.-C. Cheng. Chem. Soc. Rev. 37, 1806 (2008).

[20] E. C.-C. Cheng, W.-Y. Lo, T. K.-M. Lee, N. Zhu, V. W.-W. Yam. Inorg. Chem. 53, 3854 (2014).

[21] A. Credi, L. Prodi. Spectrochim. Acta Part A 54, 159 (1998).

[22] Caution! Perchlorate salts of metal complexes are potentially explosive. Do not dry the perchlorate salts and do measure the contents of water contained in the sample.

[23] K. Suzuki, A. Kobayashi, S. Kaneko, K. Takehira, T. Yoshihara, H. Ishida, Y. Shiina, S. Oishi, S. Tobita. Phys. Chem. Chem. Phys. 11, 9850 (2009).

[24] J. C. de Mello, H. F. Wittmann, R. H. Friend. Adv. Mat. 9, 230 (1997).

[25] H. Ishida, S. Tobita, Y. Hasegawa, R. Katoh, K. Nozaki. Coord. Chem. Rev. 254, 2449 (2010).

[26] A. Aebischer, F. Gumy, J.-C. G. Bünzli. Phys. Chem. Chem. Phys. 11, 1346 (2009).

[27] R. Katoh, K. Suzuki, A. Furube, M. Kotani, K. Tokumaru. J. Phys. Chem. C 113, 2961 (2009).

[28] N. Lu, L. M. Hight, D. R. McMillin, J.-W. Jhuo, W.-C. Chung, K.-Y. Lin, Y.-S. Wen, L.-K. Liu. Dalton Trans. 43, 2112 (2014).

[29] M. Brinkmann, G. Gadret, M. Muccini, C. Taliani, N. Masciocchi, A. Sironi. J. Am. Chem. Soc. 122, 5147 (2000).

[30] T.-S. Ahn, R. O. Al-Kaysi, A. M. Müller, K. M. Wentz, C. J. Bardeen. Rev. Sci. Instr. 78, 086105 (2007).

[31] G. Bourhill, L. O. Pålsson, I. D. W. Samuel, I. C. Sage, I. D. H. Oswald, J. P. Duignan. Chem. Phys. Lett. 336, 234 (2001).

[32] N. C. Greenham, I. D. W. Samuel, G. R. Hayes, R. T. Phillips, Y. A. R. R. Kessener, S. C. Moratti, A. B. Holmes, R. H. Friend. Chem. Phys. Lett. 241, 89 (1995).

[33] Y. Kawamura, H. Sasabe, C. Adachi. Jap. J. Appl. Phys. 43, 7729 (2004).

[34] L. O. Pålsson, A. P. Monkman. Adv. Mat. 14, 757 (2002).

[35] L. Porrès, A. Holland, L.-O. Pålsson, A. Monkman, C. Kemp, A. Beeby. J. Fluoresc. 16, 267 (2006).

[36] D. V. O'Connor, D. Phillips. Time-Correlated Single Photon Counting, Academic Press, New York (1984).

[37] W. Becker. Advanced Time-Correlated Single Photon Counting Techniques, Springer, New York (2005).

[38] J. N. Demas. Excited State Lifetime Measurements, Academic Press, New York (1983).

[39] C. Würth, M. Grabolle, J. Pauli, M. Spieles, U. Resch-Genger. Nat. Protocols 8, 1535 (2013).

[40] http://www.bipm.org/utils/common/documents/jcgm/JCGM_100_2008_E.pdf.

Note: Republication or reproduction of this report or its storage and/or dissemination by electronic means is permitted without the need for formal IUPAC or De Gruyter permission on condition that an acknowledgment, with full reference to the source, along with use of the copyright symbol $@$, the name IUPAC, the name De Gruyter, and the year of publication, are prominently visible. Publication of a translation into another language is subject to the additional condition of prior approval from the relevant IUPAC National Adhering Organization and De Gruyter. 